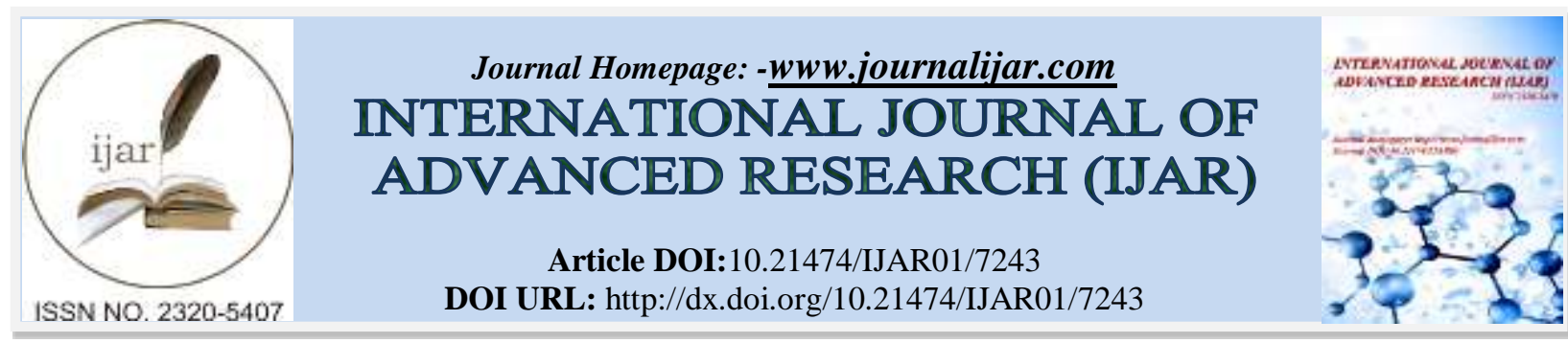

RESEARCH ARTICLE

\title{
GREY RELATION ANALYSIS BETWEEN INDUSTRIAL STRUCTURE AND ECONOMIC GROWTH BASED ON PANEL DATA.
}

Xuan Ni and Peng Lin.

School of Mathematics and Statistics, Shandong University of Technology, Zibo, China.

\section{Manuscript Info}

Manuscript History

Received: 09 April 2018

Final Accepted: 11 May 2018

Published: June 2018

Keywords:-

Industrial structure, economic growth, Grey relation analysis method, panel data

\begin{abstract}
In the process of social economic development, the change of industrial structure is closely related to the economic development. This paper selects per capita gross product and the output value of three industries of seventeen prefecture-level cities of Shandong province in China from 2012 to 2016, and discusses the relationship between industrial structure and economic growth of Shandong province through the method of grey relation theory. Results show that the correlation between economic growth and the primary industry is greater than that between economic growth and the secondary industry and the tertiary industry.

Copy Right, IJAR, 2018,. All rights reserved.
\end{abstract}

\section{Introduction:-}

The relationship between industrial structure and economic growth has been one of the most important economic discussions, and the classical theory of industrial economics has shown that industrial structural change has a strong link to economic growth, and industrial structural change is influenced by the growth of economic growth, and also the impetus for economic growth. Simon(1985) first talked about the industrial structural changes with economic growth, American economist Chenery (1995b) through research proves that the economic growth process is a group of change for national economic structure, this group of change and the growth of the national income level has the close relation. The Chinese scholar Zhou Z H(1995c) uses systematic research methods to analyze the effect of economic structure especially industrial structure change on economic growth and points out that the industrial structure is an important factor for determining economic growth. Shandong province is one of the most important provinces for China's economy, it is an effective way to improve economic growth by focusing on solving structural problems between industries and within industries as the economy enters the new normal. In order to further study the situation of industrial structure and the close relation between industrial structure adjustment and economic growth of Shandong province, this paper, uses the grey relation theory to analyze relationship between the industrial structure and economic growth of seventeen prefecture-level cities of Shandong province and offer some suggestions for the industrial structure of Shandong province.

\section{Grey relation analysis based on panel data:-}

Panel data refers to the collection of sample observation values at different time points by multiple indicators of different research objects. It has the time dimension and spatial dimension information, also known as time series cross-section data, which reflects the development level and development trend of all objects under corresponding indicators. Thus, the panel data have more overall information than a single use of cross-sectional data or time series data(2008a). 
The grey relation method was first proposed by the Deng J L(2002), which has been widely applied in the field of social sciences and natural sciences(2008b,2007,2010a,2008c,2017). It has a good application effect in the social economic field. The grey relation analysis is one of the main contents of the grey system analysis to analyze the close relationship degree between main behavior factor and relation factor in grey system and judge the main factors and minor factors of the development of the system.

Due to the limited statistical data and large grey scale in China, grey relation analysis has certain advantages compared with regression analysis, variance analysis, principal component analysis and other mathematical statistical methods in the analysis of system relation factors. Multiple statistical models require large samples and are subject to a typical distribution. But grey relation analysis applies equally to the number of samples and the irregularity of samples. Grey relational analysis is a method of statistical analysis in grey system theory. It is small sample and poor information uncertainty system of partial information known, the partial information unknown as the research object. In the incomplete information analysis to study the factors through certain data processing and find out the relation and the main influence factors, it is a method to predict the development trend of things.

Grey relational analysis is to serialize and model the grey relationship between the operating mechanism and the physical prototype that is not clear or lacks the physical prototype at all, and then establish the grey relational analysis model to make the grey relations quantify, sequence and display.

It uses the sample data of various factors as the basis to describe the strength, size and order of the factor relations with the grey relation degree.

If the sample data column reflects the basic consistency of the change of factors, the relation between them is relatively large; conversely, that is small(1998,1999,2004,1995a).Although grey relation model of research has been going on for nearly thirty years, in view of the panel data of grey relation model is still in its infancy, Zhang $\mathrm{K}(2010 \mathrm{~b})$ put forward the grey relation in the application of panel data, make up the blank in the research of grey relation in panel data. The grey relation analysis steps of the panel data are as follows:

\section{Determine the panel data analysis sequence:-}

Suppose panel data sequence $X=\left(\mathrm{X}_{0}, \mathrm{X}_{1}, \cdots, \mathrm{X}_{m}\right)$, and denote $X_{0}$ as reference sequence, $X_{1}, \ldots, X_{m}$ as comparison sequence, defined $X_{i}$ as:

$$
X_{i}=\left(\begin{array}{cccc}
X_{i}(1,1) & X_{i}(1,2) & \cdots & X_{i}(1, n) \\
\vdots & \ddots & \vdots & \vdots \\
X_{i}(N, 1) & X_{i}(N, 2) & \cdots & X_{i}(N, n)
\end{array}\right)
$$

is the panel data matrix with index $i$, and $X_{i}(s, t)$ is the value of $s t h$ object at time $t$ with index $i$, where $i=0,1,2, \cdots, m ; s=1,2, \cdots, N ; t=1,2, \cdots, n$.

\section{The calculation steps of grey relation degree of panel data:-} Dimensionless of raw data:-

Since the dimension of the various elements in the system are not necessarily the same, such data is hard to compare directly, so it need to eliminate the dimension of raw data to get comparable data sequence. The dimensionless processing of raw data can use standardized, methods of mean value and initial value, this paper uses the reciprocal of the non-zero elements in the panel data matrix, namely: $d_{i}=\frac{1}{x_{i}(1,1)}$ as the initialization operator, initialized to the matrix, namely: 


$$
\begin{gathered}
X_{i} d_{i}=\left(\begin{array}{cccc}
1 & X_{i}(1,2) d_{i} & \cdots & X_{i}(1, n) d_{i} \\
\vdots & \ddots & \vdots & \vdots \\
X_{i}(N, 1) d_{i} & X_{i}(N, 2) d_{i} & \cdots & X_{i}(N, n) d_{i}
\end{array}\right) \\
i=0,1,2, \cdots, m ; s=1,2, \cdots, N ; t=1,2, \cdots, n
\end{gathered}
$$

Panel data differential sequence, maximum difference and minimum difference:-

Reference panel data is $d_{0} X_{0}$, calculate the absolute distance of the panel data of different indicators from $d_{i} X_{i}$

to $d_{0} X_{0}, \quad$ namely: $\quad\left|x_{0}(s, t) d_{0}-x_{i}(s, t) d_{i}\right|$.And let

$$
L=\max _{i, s, t}\left|x_{0}(s, t) d_{0}-x_{i}(s, t) d_{i}\right|, S=\min _{i, s, t}\left|x_{0}(s, t) d_{0}-x_{i}(s, t) d_{i}\right|
$$

obviously, due to this paper uses the reciprocal of the non-zero elements, so $S=0, L$ realizes the maximum absolute difference of three levels in the three dimensions of research object $S$, index $i$ and time $t$.

Calculate the grey relation coefficient with the reference panel data:-

$$
\begin{gathered}
\gamma_{0 i}(s, t)=\frac{S+\rho \cdot L}{\left|x_{0}(s, t) d_{0}-x_{i}(s, t) d_{i}\right|+\rho \cdot L} \\
i=0,1,2, \cdots, m ; s=1,2, \cdots, N ; t=1,2, \cdots, n
\end{gathered}
$$

Where, $\rho$ is the resolution coefficient, and $\rho \in(0,1)$.According to Deng J L's research on grey theory, it is generally better to take $\rho$ as $0.5(2004)$, so this paper takes $\rho$ as 0.5 .

Calculate the grey relation degree with the reference panel data:-

The grey relation coefficient of different research objects at different time points is calculated by arithmetic mean, and the grey relation degree of panel data can be obtained,

$$
\begin{gathered}
\bar{\gamma}_{0 i \amalg}=\frac{1}{N \cdot n} \sum_{s=1}^{N} \sum_{t=1}^{n} \gamma_{0 i}(s, t) \\
i=0,1,2, \cdots, m ; s=1,2, \cdots, N ; t=1,2, \cdots, n
\end{gathered}
$$

The grey relation coefficient of different research objects at the same time point is calculated by arithmetic mean, and the grey relation degree of time sequence data can be obtained, namely:

$$
\begin{gathered}
\bar{\gamma}_{\mathrm{O} i t \square}=\frac{\mathbf{1}}{\boldsymbol{N}} \sum_{s=1}^{N} \gamma_{\mathrm{O} i}(\boldsymbol{s}, \boldsymbol{t}) \\
i=0,1,2, \cdots, m ; s=1,2, \cdots, N ; t=1,2, \cdots, n
\end{gathered}
$$

The grey relation coefficient of the same research object at different time points is calculated by arithmetic mean, and the grey relation degree of cross-section data can be obtained, namely:

$$
\begin{gathered}
\bar{\gamma}_{\mathrm{O} i \square s}=\frac{1}{n} \sum_{n=1}^{n} \gamma_{\mathrm{O} i}(s, t) \\
i=0,1,2, \cdots, m ; s=1,2, \cdots, N ; t=1,2, \cdots, n
\end{gathered}
$$

The empirical research:-

Data source:-

This paper selects panel data of seventeen prefecture-level cities in Shandong province from2012 to 2016, with per capita gross product to reflect the indicators of economic growth, the unit is yuan, with the output value of primary 
industry, secondary industry and tertiary industry to reflect the industrial structure, the unit is billion yuan, data from statistical yearbook of Shandong(2013-2017).

Empirical analysis results:-

The initial value after dimensionless treatment are shown in table 1,2.

Table 1:-Initialized panel data

\begin{tabular}{|c|c|c|c|c|c|c|c|c|c|c|}
\hline $\begin{array}{l}\text { Classifica- } \\
\text { tion }\end{array}$ & Year & Jinan & Qingdao & Zibo & Zaozhuang & Dongying & Yantai & Weifang & Jining & Taian \\
\hline \multirow{5}{*}{$\begin{array}{l}\text { per capita } \\
\text { gross } \\
\text { product }\end{array}$} & 2012 & 1.000 & 1.191 & 1.121 & 0.652 & 2.094 & $\begin{array}{l}1.090 \\
\end{array}$ & 0.629 & 0.564 & 0.664 \\
\hline & 2013 & 1.080 & 1.293 & 1.194 & 0.696 & 2.252 & 1.157 & 0.690 & 0.616 & 0.724 \\
\hline & 2014 & 1.182 & 1.390 & 1.260 & 0.747 & 2.361 & 1.235 & 0.746 & 0.665 & 0.775 \\
\hline & 2015 & 1.237 & 1.476 & 1.285 & 0.759 & 2.361 & 1.325 & 0.804 & 0.699 & 0.813 \\
\hline & 2016 & 1.310 & 1.575 & 1.362 & 0.792 & 2.362 & 1.417 & 0.854 & 0.744 & 0.850 \\
\hline \multirow{5}{*}{$\begin{array}{l}\text { Primary } \\
\text { industry }\end{array}$} & 2012 & 1.000 & 1.283 & 0.489 & 0.526 & 0.413 & 1.492 & 1.544 & 1.471 & 0.921 \\
\hline & 2013 & 1.095 & 1.346 & 0.531 & 0.562 & 0.434 & 1.613 & 1.645 & 1.615 & 0.990 \\
\hline & 2014 & 1.148 & 1.382 & 0.554 & 0.584 & 0.458 & 1.687 & 1.729 & 1.706 & 1.029 \\
\hline & 2015 & 1.207 & 1.439 & 0.573 & 0.609 & 0.466 & 1.743 & 1.800 & 1.796 & 1.064 \\
\hline & 2016 & 1.255 & 1.467 & 0.596 & 0.641 & 0.482 & 1.848 & 1.879 & 1.900 & 1.111 \\
\hline \multirow{5}{*}{$\begin{array}{l}\text { Secondary } \\
\text { industry }\end{array}$} & 2012 & 1.000 & 1.755 & 1.084 & 0.511 & 1.097 & 1.540 & 1.118 & 0.863 & 0.666 \\
\hline & 2013 & 1.081 & 1.884 & 1.120 & 0.526 & 1.134 & 1.571 & 1.158 & 0.900 & 0.697 \\
\hline & 2014 & 1.167 & 2.007 & 1.160 & 0.555 & 1.179 & 1.641 & 1.224 & 0.962 & 0.738 \\
\hline & 2015 & 1.190 & 2.077 & 1.150 & 0.552 & 1.151 & 1.715 & 1.285 & 0.978 & 0.754 \\
\hline & 2016 & 1.222 & 2.147 & 1.195 & 0.566 & 1.116 & 1.786 & 1.321 & 1.006 & 0.766 \\
\hline \multirow{5}{*}{$\begin{array}{l}\text { Tertiary } \\
\text { industry }\end{array}$} & 2012 & 1.000 & 1.369 & 0.510 & 0.221 & 0.295 & 0.735 & 0.557 & 0.438 & 0.392 \\
\hline & 2013 & 1.094 & 1.537 & 0.573 & 0.256 & 0.361 & 0.827 & 0.673 & 0.516 & 0.455 \\
\hline & 2014 & 1.232 & 1.704 & 0.629 & 0.290 & 0.394 & 0.917 & 0.757 & 0.576 & 0.502 \\
\hline & 2015 & 1.335 & 1.879 & 0.672 & 0.309 & 0.422 & 1.026 & 0.851 & 0.636 & 0.546 \\
\hline & 2016 & 1.474 & 2.097 & 0.745 & 0.338 & 0.457 & 1.147 & 0.952 & 0.716 & 0.593 \\
\hline
\end{tabular}

Table 2:-Initialized panel data

\begin{tabular}{|c|c|c|c|c|c|c|c|c|c|}
\hline $\begin{array}{l}\text { Classifica- } \\
\text { tion }\end{array}$ & Year & Weihai & Rizhao & Laiwu & Linyi & Dezhou & Liaocheng & Binzhou & Heze \\
\hline \multirow{5}{*}{$\begin{array}{l}\text { per capita } \\
\text { gross } \\
\text { product }\end{array}$} & 2012 & 1.203 & 0.689 & 0.694 & 0.429 & 0.572 & 0.527 & 0.757 & 0.309 \\
\hline & 2013 & 1.311 & 0.760 & 0.711 & 0.474 & 0.627 & 0.577 & 0.817 & 0.353 \\
\hline & 2014 & 1.431 & 0.811 & 0.739 & 0.504 & 0.657 & 0.612 & 0.858 & 0.381 \\
\hline & 2015 & 1.540 & 0.837 & 0.711 & 0.528 & 0.692 & 0.644 & 0.881 & 0.408 \\
\hline & 2016 & 1.645 & 0.898 & 0.742 & 0.559 & 0.732 & 0.686 & 0.918 & 0.431 \\
\hline \multirow{5}{*}{$\begin{array}{l}\text { Primary } \\
\text { industry }\end{array}$} & 2012 & 0.712 & 0.465 & 0.175 & 1.152 & 0.966 & 1.019 & 0.749 & 0.953 \\
\hline & 2013 & 0.780 & 0.498 & 0.194 & 1.248 & 1.017 & 1.112 & 0.799 & 0.994 \\
\hline & 2014 & 0.821 & 0.526 & 0.209 & 1.310 & 1.070 & 1.203 & 0.834 & 1.031 \\
\hline & 2015 & 0.859 & 0.556 & 0.208 & 1.370 & 1.122 & 1.251 & 0.860 & 1.068 \\
\hline & 2016 & 0.907 & 0.581 & 0.218 & 1.419 & 1.171 & 1.337 & 0.918 & 1.110 \\
\hline \multirow{5}{*}{$\begin{array}{l}\text { Secondary } \\
\text { industry }\end{array}$} & 2012 & 0.645 & 0.374 & 0.188 & 0.755 & 0.624 & 0.612 & 0.539 & 0.503 \\
\hline & 2013 & 0.660 & 0.399 & 0.188 & 0.817 & 0.656 & 0.652 & 0.567 & 0.574 \\
\hline & 2014 & 0.699 & 0.419 & 0.193 & 0.850 & 0.674 & 0.673 & 0.591 & 0.614 \\
\hline & 2015 & 0.734 & 0.420 & 0.178 & 0.870 & 0.701 & 0.702 & 0.593 & 0.654 \\
\hline & 2016 & 0.755 & 0.440 & 0.182 & 0.896 & 0.724 & 0.730 & 0.590 & 0.677 \\
\hline \multirow{5}{*}{$\begin{array}{l}\text { Tertiary } \\
\text { industry }\end{array}$} & 2012 & 0.348 & 0.196 & 0.085 & 0.482 & 0.298 & 0.269 & 0.288 & 0.219 \\
\hline & 2013 & 0.411 & 0.230 & 0.092 & 0.550 & 0.357 & 0.314 & 0.327 & 0.262 \\
\hline & 2014 & 0.470 & 0.255 & 0.100 & 0.609 & 0.390 & 0.347 & 0.352 & 0.295 \\
\hline & 2015 & 0.521 & 0.274 & 0.103 & 0.662 & 0.425 & 0.378 & 0.378 & 0.330 \\
\hline & 2016 & 0.582 & 0.308 & 0.113 & 0.739 & 0.472 & 0.423 & 0.419 & 0.370 \\
\hline
\end{tabular}


With per capita gross product as the reference sequence, calculate absolute distance between three industry and reference sequence respectively are shown in table3, 4.

Table3:-Absolute distance between three industry and reference sequence

\begin{tabular}{|l|c|c|c|c|c|c|c|c|c|c|}
\hline $\begin{array}{l}\text { Classifica- } \\
\text { tion }\end{array}$ & Year & Jinan & Qingdao & Zibo & Zaozhuang & Dongying & Yantai & Weifang & Jining & Taian \\
\hline \multirow{4}{*}{$\begin{array}{l}\text { Primary } \\
\text { industry }\end{array}$} & 2012 & 0.000 & 0.092 & 0.632 & 0.126 & 1.681 & 0.402 & 0.915 & 0.907 & 0.257 \\
\cline { 2 - 11 } & 2013 & 0.015 & 0.053 & 0.662 & 0.134 & 1.818 & 0.455 & 0.955 & 0.998 & 0.265 \\
\cline { 2 - 11 } & 2014 & 0.034 & 0.008 & 0.706 & 0.163 & 1.903 & 0.451 & 0.983 & 1.040 & 0.254 \\
\cline { 2 - 11 } & 2015 & 0.030 & 0.037 & 0.712 & 0.149 & 1.895 & 0.419 & 0.996 & 1.097 & 0.250 \\
\cline { 2 - 11 } & 2016 & 0.056 & 0.109 & 0.766 & 0.151 & 1.880 & 0.432 & 1.026 & 1.156 & 0.261 \\
\hline \multirow{3}{*}{$\begin{array}{l}\text { Secondary } \\
\text { industry }\end{array}$} & 2012 & 0.000 & 0.565 & 0.037 & 0.140 & 0.997 & 0.450 & 0.489 & 0.299 & 0.002 \\
\cline { 2 - 11 } & 2013 & 0.001 & 0.591 & 0.074 & 0.171 & 1.118 & 0.414 & 0.468 & 0.283 & 0.027 \\
\cline { 2 - 11 } & 2014 & 0.015 & 0.617 & 0.101 & 0.192 & 1.183 & 0.405 & 0.478 & 0.297 & 0.038 \\
\cline { 2 - 11 } & 2015 & 0.047 & 0.601 & 0.135 & 0.207 & 1.210 & 0.390 & 0.481 & 0.280 & 0.059 \\
\cline { 2 - 10 } industry & 2016 & 0.088 & 0.571 & 0.167 & 0.225 & 1.246 & 0.369 & 0.467 & 0.262 & 0.084 \\
\cline { 2 - 10 } & 2012 & 0.000 & 0.178 & 0.611 & 0.430 & 1.799 & 0.355 & 0.072 & 0.126 & 0.273 \\
\cline { 2 - 10 } & 2013 & 0.014 & 0.244 & 0.621 & 0.440 & 1.891 & 0.330 & 0.017 & 0.100 & 0.269 \\
\cline { 2 - 10 } & 2015 & 0.050 & 0.314 & 0.632 & 0.458 & 1.967 & 0.318 & 0.010 & 0.090 & 0.273 \\
\cline { 2 - 10 } & 2016 & 0.163 & 0.522 & 0.617 & 0.454 & 1.905 & 0.270 & 0.099 & 0.028 & 0.257 \\
\hline
\end{tabular}

Table 4:-Absolute distance between three industry and reference sequence

\begin{tabular}{|l|c|c|c|c|c|c|c|c|c|}
\hline $\begin{array}{l}\text { Classifica- } \\
\text { tion }\end{array}$ & Year & Weihai & Rizhao & Laiwu & Linyi & Dezhou & Liaocheng & Binzhou & Heze \\
\hline $\begin{array}{l}\text { Primary } \\
\text { industry }\end{array}$ & 2012 & 0.491 & 0.224 & 0.519 & 0.723 & 0.394 & 0.493 & 0.008 & 0.644 \\
\cline { 2 - 10 } & 2013 & 0.531 & 0.262 & 0.518 & 0.774 & 0.390 & 0.535 & 0.018 & 0.641 \\
\cline { 2 - 10 } & 2014 & 0.611 & 0.285 & 0.531 & 0.805 & 0.412 & 0.591 & 0.024 & 0.650 \\
\cline { 2 - 10 } & 2015 & 0.681 & 0.281 & 0.503 & 0.842 & 0.430 & 0.607 & 0.021 & 0.660 \\
\cline { 2 - 10 } & 2016 & 0.738 & 0.317 & 0.524 & 0.860 & 0.439 & 0.651 & 0.000 & 0.679 \\
\hline \multirow{4}{*}{$\begin{array}{l}\text { Secondary } \\
\text { industry }\end{array}$} & 2012 & 0.558 & 0.315 & 0.506 & 0.326 & 0.052 & 0.085 & 0.218 & 0.194 \\
\cline { 2 - 10 } & 2013 & 0.651 & 0.361 & 0.523 & 0.343 & 0.029 & 0.075 & 0.251 & 0.221 \\
\cline { 2 - 10 } & 2014 & 0.732 & 0.393 & 0.547 & 0.346 & 0.017 & 0.062 & 0.267 & 0.233 \\
\cline { 2 - 10 } & 2015 & 0.806 & 0.417 & 0.533 & 0.343 & 0.009 & 0.058 & 0.288 & 0.246 \\
\hline \multirow{4}{*}{$\begin{array}{l}\text { Tertiary } \\
\text { industry }\end{array}$} & 2016 & 0.890 & 0.458 & 0.560 & 0.337 & 0.008 & 0.044 & 0.328 & 0.247 \\
\cline { 2 - 10 } & 2012 & 0.855 & 0.494 & 0.609 & 0.052 & 0.274 & 0.258 & 0.469 & 0.090 \\
\cline { 2 - 10 } & 2014 & 0.900 & 0.530 & 0.619 & 0.077 & 0.270 & 0.263 & 0.490 & 0.091 \\
\cline { 2 - 10 } & 2015 & 1.018 & 0.556 & 0.639 & 0.104 & 0.267 & 0.265 & 0.505 & 0.086 \\
\cline { 2 - 10 } & 2016 & 1.063 & 0.590 & 0.608 & 0.134 & 0.268 & 0.267 & 0.503 & 0.078 \\
\hline
\end{tabular}

Given the grey resolution coefficient $\rho=0.5$, the grey relation coefficient of panel data between three industry and per capita gross product are shown in table 5,6.

Table5:-Relation coefficient of panel data

\begin{tabular}{|c|c|c|c|c|c|c|c|c|c|c|}
\hline $\begin{array}{c}\text { Classifica- } \\
\text { tion }\end{array}$ & Year & Jinan & Qingdao & Zibo & Zaozhuang & Dongying & Yantai & Weifang & Jining & Taian \\
\hline \multirow{3}{*}{$\begin{array}{c}\text { Primary } \\
\text { industry }\end{array}$} & 2012 & 1.000 & 0.914 & 0.609 & 0.887 & 0.369 & 0.710 & 0.518 & 0.520 & 0.793 \\
\cline { 2 - 11 } & 2013 & 0.985 & 0.949 & 0.598 & 0.880 & 0.351 & 0.684 & 0.507 & 0.496 & 0.788 \\
\cline { 2 - 11 } & 2014 & 0.967 & 0.992 & 0.582 & 0.858 & 0.341 & 0.686 & 0.500 & 0.486 & 0.795 \\
\cline { 2 - 11 } & 2015 & 0.971 & 0.964 & 0.580 & 0.868 & 0.342 & 0.701 & 0.497 & 0.473 & 0.797 \\
\cline { 2 - 11 } & 2016 & 0.946 & 0.901 & 0.562 & 0.867 & 0.343 & 0.695 & 0.489 & 0.460 & 0.790 \\
\hline \multirow{2}{*}{$\begin{array}{c}\text { Secondary } \\
\text { industry }\end{array}$} & 2012 & 1.000 & 0.635 & 0.963 & 0.875 & 0.497 & 0.686 & 0.668 & 0.767 & 0.998 \\
\cline { 2 - 11 } & 2013 & 0.999 & 0.625 & 0.930 & 0.852 & 0.468 & 0.704 & 0.678 & 0.776 & 0.973 \\
\cline { 2 - 11 } & 20.985 & 0.614 & 0.907 & 0.837 & 0.454 & 0.708 & 0.673 & 0.768 & 0.963 \\
\hline
\end{tabular}




\begin{tabular}{|c|c|c|c|c|c|c|c|c|c|c|}
\hline & 2015 & 0.954 & 0.621 & 0.879 & 0.826 & 0.448 & 0.716 & 0.671 & 0.779 & 0.943 \\
\cline { 2 - 11 } & 2016 & 0.918 & 0.633 & 0.855 & 0.814 & 0.441 & 0.727 & 0.678 & 0.790 & 0.922 \\
\hline \multirow{3}{*}{$\begin{array}{c}\text { Tertiary } \\
\text { industry }\end{array}$} & 2012 & 1.000 & 0.847 & 0.617 & 0.696 & 0.353 & 0.735 & 0.932 & 0.886 & 0.783 \\
\cline { 2 - 10 } & 2013 & 0.986 & 0.802 & 0.613 & 0.691 & 0.342 & 0.749 & 0.983 & 0.908 & 0.785 \\
\cline { 2 - 11 } & 2014 & 0.951 & 0.758 & 0.609 & 0.682 & 0.333 & 0.755 & 0.989 & 0.916 & 0.783 \\
\cline { 2 - 10 } & 2015 & 0.910 & 0.709 & 0.616 & 0.686 & 0.337 & 0.767 & 0.954 & 0.940 & 0.786 \\
\cline { 2 - 10 } & 2016 & 0.858 & 0.653 & 0.614 & 0.684 & 0.341 & 0.785 & 0.909 & 0.973 & 0.793 \\
\hline
\end{tabular}

Table 6:-Relation coefficient of panel data

\begin{tabular}{|c|c|c|c|c|c|c|c|c|c|}
\hline Classification & Year & Weihai & Rizhao & Laiwu & Linyi & Dezhou & Liaocheng & Binzhou & Heze \\
\hline \multirow{3}{*}{$\begin{array}{c}\text { Primary } \\
\text { industry }\end{array}$} & 2012 & 0.667 & 0.815 & 0.654 & 0.576 & 0.714 & 0.666 & 0.992 & 0.604 \\
\cline { 2 - 10 } & 2013 & 0.649 & 0.789 & 0.655 & 0.560 & 0.716 & 0.648 & 0.982 & 0.605 \\
\cline { 2 - 10 } & 2014 & 0.617 & 0.775 & 0.649 & 0.550 & 0.705 & 0.625 & 0.976 & 0.602 \\
\cline { 2 - 10 } & 2015 & 0.591 & 0.778 & 0.662 & 0.539 & 0.696 & 0.619 & 0.979 & 0.599 \\
\cline { 2 - 10 } & 2016 & 0.571 & 0.756 & 0.652 & 0.533 & 0.691 & 0.602 & 1.000 & 0.592 \\
\hline \multirow{3}{*}{$\begin{array}{c}\text { Secondary } \\
\text { industry }\end{array}$} & 2012 & 0.638 & 0.757 & 0.660 & 0.751 & 0.950 & 0.920 & 0.819 & 0.836 \\
\cline { 2 - 10 } & 2013 & 0.602 & 0.732 & 0.653 & 0.741 & 0.972 & 0.929 & 0.797 & 0.817 \\
\cline { 2 - 10 } & 2014 & 0.573 & 0.715 & 0.643 & 0.740 & 0.983 & 0.941 & 0.787 & 0.808 \\
\cline { 2 - 10 } & 2015 & 0.550 & 0.702 & 0.648 & 0.742 & 0.991 & 0.945 & 0.774 & 0.800 \\
\cline { 2 - 10 } industry & 2016 & 0.525 & 0.682 & 0.637 & 0.745 & 0.992 & 0.957 & 0.750 & 0.800 \\
\cline { 2 - 10 } & 2012 & 0.535 & 0.666 & 0.617 & 0.950 & 0.782 & 0.792 & 0.677 & 0.916 \\
\cline { 2 - 10 } & 2013 & 0.522 & 0.650 & 0.614 & 0.928 & 0.785 & 0.789 & 0.667 & 0.915 \\
\cline { 2 - 10 } & 2014 & 0.506 & 0.639 & 0.606 & 0.904 & 0.786 & 0.788 & 0.661 & 0.920 \\
\cline { 2 - 9 } & 2015 & 0.491 & 0.636 & 0.618 & 0.880 & 0.786 & 0.787 & 0.662 & 0.927 \\
\cline { 2 - 9 } & 2016 & 0.481 & 0.625 & 0.610 & 0.845 & 0.791 & 0.789 & 0.664 & 0.942 \\
\hline
\end{tabular}

The grey relational degree of panel data are shown in table 7 .

Table 7:-Grey relation degree of panel data \begin{tabular}{|l|l}
\hline per capita gross product and primary & Per capita gross product and
\end{tabular} industry secondary industry

gross product and tertiary industry 0.596 0.564 0.573

The grey relation degree of panel data shows that the grey relation degree between economic growth of Shandong province and three industry is as follows: primary industry> tertiary industry> secondary industry, therefore economic growth of Shandong province mainly depends on primary industry.

The grey relation degree of time sequence data are shown in table 8 .

Table 8:-Grey relation degree of time sequence data

\begin{tabular}{|c|c|c|c|c|c|}
\hline & 2012 & 2013 & 2014 & 2015 & 2016 \\
\hline $\begin{array}{c}\text { per capita gross } \\
\text { product and } \\
\text { primary industry }\end{array}$ & 0.592 & 0.595 & 0.596 & 0.600 & 0.594 \\
\hline $\begin{array}{c}\text { per capita gross } \\
\text { product and } \\
\text { secondary industry }\end{array}$ & 0.558 & 0.562 & 0.565 & 0.567 & 0.569 \\
\hline $\begin{array}{c}\text { per capita gross } \\
\text { product and tertiary } \\
\text { industry }\end{array}$ & 0.564 & 0.572 & 0.574 & 0.576 & 0.578 \\
\hline
\end{tabular}

The economic growth of Shandong province depends largely on the primary industry. It is worth noting that the relation between the economic growth and the tertiary industry of Shandong province shows a upward trend year by year.

The grey relation degree of cross-section data are shown in table 9. 
Table 9:-Grey relation degree of cross-section data

\begin{tabular}{|c|c|c|c|}
\hline region & $\begin{array}{c}\text { per capita gross product } \\
\text { and primary industry }\end{array}$ & $\begin{array}{c}\text { per capita gross product } \\
\text { and secondary industry }\end{array}$ & $\begin{array}{c}\text { per capita gross product } \\
\text { and tertiary industry }\end{array}$ \\
\hline Jinan & 0.974 & 0.971 & 0.941 \\
\hline Qingdao & 0.944 & 0.626 & 0.754 \\
\hline Zibo & 0.586 & 0.907 & 0.614 \\
\hline Zaozhuang & 0.872 & 0.841 & 0.688 \\
\hline Dongying & 0.349 & 0.462 & 0.341 \\
\hline Yantai & 0.695 & 0.708 & 0.758 \\
\hline Weifang & 0.502 & 0.674 & 0.953 \\
\hline Jining & 0.487 & 0.776 & 0.925 \\
\hline Taian & 0.793 & 0.960 & 0.786 \\
\hline Weihai & 0.619 & 0.578 & 0.507 \\
\hline Rizhao & 0.783 & 0.718 & 0.643 \\
\hline Laiwu & 0.655 & 0.648 & 0.613 \\
\hline Linyi & 0.552 & 0.744 & 0.901 \\
\hline Dezhou & 0.704 & 0.978 & 0.786 \\
\hline Lioacheng & 0.632 & 0.938 & 0.789 \\
\hline Binzhou & 0.986 & 0.785 & 0.666 \\
\hline Heze & 0.600 & 0.812 & 0.924 \\
\hline
\end{tabular}

The results showed that seventeen prefecture-level cities of Shandong province can be divided into five types: primary industry > secondary industry >tertiary industry (Jinan, Zaozhuang, Weihai, Rizhao, Binzhou, Laiwu ); secondary industry >tertiary industry > primary industry (Zibo, Dezhou, Liaocheng); Secondary industry > primary industry $>$ tertiary industry (Dongying, Taian); tertiary industry $>$ Secondary industry $>$ primary industry (Yantai, Weifang, Jining, Linyi, Heze); primary industry >tertiary industry > secondary industry (Qingdao), on the whole, the economic growth of various cities is still significantly tilted towards the primary industry.

\section{Conclusions and suggestions:-}

Based on the above empirical research on industrial structure and economic growth of Shandong province, the results show that: primary industry has certain grey relation degree to the economic growth of Shandong province and it is slightly higher than secondary industry and tertiary industry, it means that primary industry has a relatively large contribution to economic growth.

In order to promote the development of Shandong province's industrial structure and optimize Shandong province's industrial structure, relevant suggestions are put forward:

In primary industry, Shandong province should further accelerate transformation of the mode of agricultural development in the process of industrial structure adjustment as a big agricultural province of China. Increase the added value of products, and promoting agricultural products by relies mainly on the primary products to processing products, deep processing of agricultural products, extend the industrial chain, improve the added value of agricultural products, develop the marine products, cereals, oils and processed agricultural products, encouraging agricultural products brand, thus expand the market.

In secondary industry, the way of transforming economic growth, developing circular economy, lower energy efficiency and new development roads, vigorously developing new and high technology industries, and increasing the proportion of high-tech industry in the total value of industry. To promote the industrial development, guarantee the improvement of the traditional industry technology, technology, management and operation on the basis of sustainable development, but also to promote the development of new industries, promote the development of new and high technology industries, set up the leading industry of the province, made ready a number of national wellknown international brands, and promote the development of secondary industries on the basis of improving economic benefits.

In the tertiary industry, the development of tertiary industry is not only conducive to the optimization and upgrading of industrial structure and guarantee the healthy development of national economy. It is more important that tertiary industry be able to absorb substantial amounts of employment. It is an important way to solve rural surplus labor, 
therefore, Shandong province should take active measures to develop the tertiary industry. Focus on the modern service industry, actively guiding, deepening reform, promoting innovation in management mechanisms, and promoting the healthy and orderly development of the service industry. Paying attention to overall planning, distinguish priorities and lay particular emphasis at the same time.

\section{References:-}

1. Bai Z L. (2008a): Econometric Analysis of Panel Data. Nankai University Press.

2. Chen H T, Zhou D Q. (2008b): Study on the time difference between prices of China and international oil futures based on grey relativity theory. Systems Engineering Theory Practice, 28(3): 166-170.

3. Deng J L.(2002): Grey theory basis. Huazhong University of Science and Technology University Press.

4. Dang Y G, Zhu X Y, et al. (2017): Panel data clustering method based on grey correlation degree and its application in air pollution analysis. Control and Decision,32(12): 2227-2232.

5. Deng J L. (1998): Grey group decision in grey rational space. The Journal of Grey System, 10(3): 177-182.

6. Deng J L. (2004): Figure on difference information space in grey relational analysis. (2004): The Journal of Grey System, 16(2): 96-100.

7. Deng J L. (1995a): Spread of grey relational space. The Journal of Grey System, 7(3):96-100.

8. H. Chenery, S. Robinson, M. (1995b): Comparative Study on Industrialization and Economic Growth. Shanghai People's Publishing House

9. Hu Q Z Z, Zhang W H. (2007): Grey related degree model for the optimization of urban public transport network. Journal of Systems Engineering, 22(6):607-612.

10. Qian W Y, Dang Y G, Xiong P P, et al.(2008c): TOPSIS based on grey correlation method and its application. Systems Engineering, 27(8): 124-126.

11. Simon Kuznets. (1985):Economic Growth of Nations: Total output and production structure. The Commercial Press.

12. Shi H X, Liu S F, Fang Z G, et al.(2010a): Grey amplitude incidence model. Systems Engineering Theory Practice, 30(10): 1828-1833.

13. Wen K L, Chang T C. (1999): Data preprocessing for grey relational analysis source. The Journal of Grey System, 11(2): 139-142.

14. Zhou Z H.(1995c): Structural Effects of Modern Economic Growth. Shanghai People's Publishing House.

15. Zhang K, Liu S F. (2010b): Extended clusters of grey incidence for panel data and its application. Systems Engineering,30(7): 1253-1259. 